\title{
Childhood Mortality in Kenya: Survival Analysis of 2014 Kenya Demographic and Health Survey Data
}

\author{
EIda Naliaka Watulo*, Anthony Kibira Wanjoya \\ Email address: \\ elda.watulo@gmail.com (E. N. Watulo), awanjoya@gmail.com (A. K. Wanjoya) \\ ${ }^{*}$ Corresponding author
}

Department of Statistics and Actuarial Science, Jomo Kenyatta University of Agriculture and Technology, Nairobi, Kenya

\section{To cite this article:}

Elda Naliaka Watulo, Anthony Kibira Wanjoya. Childhood Mortality in Kenya: Survival Analysis of 2014 Kenya Demographic and Health Survey Data. International Journal of Statistical Distributions and Applications. Vol. 7, No. 2, 2021, pp. 57-71.

doi: $10.11648 /$ j.jissd.20210702.14

Received: June 11, 2021; Accepted: June 23, 2021; Published: June 28, 2021

\begin{abstract}
Childhood mortality is still a public health issue in Sub-Saharan Africa, with Kenya being among the countries that experience the highest rate of children dying before reaching the age of five. Under-5 child mortality (U5CM) is heavily influenced by demographic, environmental, and socio-economic factors. The study aimed to examine the risk factors of under-5 child mortality in Kenya. The data were based on birth histories from the Kenya Demographic and Health Surveys (KDHS) conducted in 2014. The relative contribution of factors such as the mother's education, mother's occupation, household wealth, place of residence, region, and sex of the child to the variability in the under-five child mortality was assessed using Kaplan-Meier and Cox hazard methods. The outcome variable for the study was the child's survival before the age of 5 and age at death. All children born in the period between 2009 and $2014(\mathrm{n}=83,591)$ were included in the study. Within the observation period, a total of 6,123 child deaths were recorded. The under-5 mortality rate in Kenya was strongly associated with the mother's education, region, place of residence, preceding birth interval, birth order, the total number of children ever born, mother's occupation, and type of toilet facility. The results indicated that a child born in Nyanza is twice more likely to die than that born in the Central region of Kenya. Male children had a higher risk of dying before the age of five than their female counterparts. The risk of experiencing U5CM increased among children born in rural areas compared to those born in urban areas. The study findings provide evidence in support of prioritizing interventions aiming at improving maternal and child healthcare. The findings also suggest that programs aimed at empowering women and boosting health knowledge among mothers should be scaled up. Furthermore, implementing socio-economic development interventions that reduce regional disparities is a recommendation that the central government should consider. Finally, national and local governments should commit resources to guarantee that modern contraceptives are available and used to increase child spacing.
\end{abstract}

Keywords: Under-Five Child Mortality, Survival Models, Cox-proportional Hazard, Kaplan-Meier,

Kenya Demographic and Health Survey

\section{Introduction}

Child mortality indicates the overall health and well-being of a nation. Besides reflecting the magnitude of the health issues that are directly responsible for the deaths of children, such as malnutrition and diarrheal infections, it also indicates the net effect of other factors such as pre-and postnatal care of mothers and children, as well as the environmental conditions in which the child is exposed. Reduced child mortality is one of the key objectives of the fourth Millennium Development Goals, MDG4, and the third Sustainable Development Goal,
SDG 3. Under-five deaths in Kenya today stem from problems long since solved in developed countries, such as protection against malaria-causing mosquitoes, lack of access to clean water, and poor sanitation [1].

According to the 2013 UN-IGME report, the under-five mortality rate declined to 53 deaths per 1,000 live births in 2009, down from 78 deaths per 1,000 live births in 1999, with the developing nations, especially Sub-Saharan African countries, accounting for the majority of these deaths [1]. Latin America and the Caribbean, Northern Africa, Eastern Asia, Western Asia, and South-Eastern Asia are among the 
countries that saw the most substantial reduction (of over $50 \%$ ) in child deaths. Sub-Saharan Africa (SSA) saw the lowest reduction, with a 39 percent drop. Different factors contribute to these regional disparities, including differences in environmental, social, and health conditions.

The same report [1] indicates that one out of every nine children in Sub-Saharan Africa dies before reaching the age of five. Between 1965 and 1980, Kenya's under-five mortality rate decreased drastically [2], which was twice that of an average Sub-Saharan country at the time. Several Kenyan research institutions found that the reduction in U5MR had not just slowed but reversed throughout the 1990s, based on data from the Kenya Demographic Health Survey. An analysis of the 2008/9 Kenya Demographic Health Survey data revealed a reversal in mortality rates in the late 1990s and early 2000s, with the rate dropping by $36 \%$ (from 115 deaths to 74 deaths per 1000 live births). This significant decrease in the under-five mortality rates was attributed to the government's efforts to improve health services [3].

Using data from the 2014 Kenya Demographic Health Survey, the goal of this study is to determine the risk factors of under-five child mortality in Kenya. The study's justification is that it provides new data on the causes and trends of child mortality, which may be used to develop and implement health policies and interventions that support child survival beyond five years. This study is also important because there have been few studies on this topic in Kenya, and none have used the 2014 Kenya Demographic and Health Survey (KDHS), which is the most recent health survey data. The findings of this study could also be a reference for dealing with future causes of under-five mortality. Therefore, the insights from this study should be extremely helpful to the Ministry of Health and other health-care stakeholders in developing and implementing child health interventions in Kenya and other Sub-Saharan African countries.

\section{Related Work}

In their demographic researches, Schultz [4], Mosley and Chen [5] divided factors affecting under-5 child mortality into social-demographic, socio-economic, and environmental factors.

\subsection{Social-Demographic Factors}

According to Mosley and Chen [5], birth order, sex of a child, and mother's age at first birth are among the socio-demographic factors that affect child mortality. Similar studies were conducted by Rutstein [6] and Davanzo et al. [7].

In Kenya, childhood mortality patterns caused by mothers' age are often U-shaped. Children born to mothers who are younger (under the age of 20) or older (beyond the age of 40) have greater mortality rates than children born to mothers who are between the ages of 20 and 40 [8]. Kibet [8] observed that younger mothers are unskilled in caring for their children. $\mathrm{He}$ further adds that a child born to a younger mother is more likely to be malnourished, underweight, and anemic, all of which increase the chance of death.
Both infant and child mortality have been found to be strongly influenced by birth intervals.

Short birth intervals have been linked to poor birth outcomes, greater pregnant morbidity rates, and higher infant and child mortality rates [9]. Kumar and File [10] examined selected bio-demographic, socioeconomic, and maternal health care determinants of child mortality in Ethiopia using cross-tabulation techniques on the Ethiopian DHS 2005 survey data. According to the findings, birth interval is one of the social demographic factors that has a significant impact on child mortality.

Mutunga [11] assessed the effect of environmental and social demographic determinants of infant and child mortality in the Kenyan urban areas using data from the Kenyan Demographic Health Survey 2003. The findings showed that children with birth intervals of less than two years had a greater risk of death.

Many studies, such as Mosley and Chen's [5], have found a strong association between birth order and child mortality. First-born children, according to these studies, are more likely to die, maybe due to their mother's age or birth complications. The greater risk of child mortality among first births may be due to the fact that such births mostly occur among younger mothers who are inexperienced in caring for children.

Several studies have related a child's gender to child mortality, with boys having a higher risk of dying before reaching the age of five than their female counterparts. Experts attribute this to variations in biological and genetic makeup between male and female children, with male children being naturally weaker and more susceptible to illnesses and early mortality.

The length of time a child is breastfed has also been linked to child mortality. Breast milk is nutritious and vital for a child's growth since it contains nutrients, hormones, antioxidants, and antibodies that are essential for a child's life. Children who are breastfed exclusively for the first six months of their lives and continue nursing until they are at least two years old have a stronger immune system than those who do not [12]. Infants are born with considerable immunity to illnesses thanks to the antibodies passed down through the placenta from their mothers. Breast milk continues to provide additional antibodies after birth, ensuring that a child remains strong and healthy. The immunity weakens as the children are introduced to new meals.

\subsection{Socioeconomic Factors}

Socioeconomic factors that influence child mortality include mother's level of education, place of residence, mother's employment status, and region.

Different studies have shown a strong association between child mortality and the mother's employment status. The amount of money a mother earns from her job is a factor in a child's survival prospects since it impacts how she can meet the basic needs of her family. Mothers who are employed have the financial means to provide their children with high-quality health care, improving their chances of survival.

Mothers who work away from home, on the other hand, may be unable to provide the essential child care, particularly 
if their income is low. In this situation, it is the mother's absence from caring for her child, not her employment, that has a negative impact on the child's survival, since lack of proper breastfeeding during the first months after birth reduces the chances of a child's survival [13]. For mothers who work from home, the effects may be different.

One of the factors that determine a child's health and death is where they live. Children living in urban areas with safe drinking water, good sanitation, and improved health care facilities have reduced mortality rates, according to Stalling [14]. According to Woldemicael [15], the place where a mother lives influences her access to education as well as the availability of good sanitation, health care, and clean water, all of which impact a child's chances of survival.

One of the prerequisites for a country's socioeconomic success is education; a well-educated people is wealthier and healthier. Higher educational levels, according to Mutunga [16], are usually linked to lower child death rates because education exposes mothers to information on improved nutrition, pediatric disease treatments, and the use of family planning methods to space births.

Goro [17] examined the factors affecting child mortality in Ghana's three northern regions by analyzing data from the DHS surveys conducted in 1993, 1998, and 2003 using a multivariate logistic regression model. He discovered that marital status, birth order, and mother's level of educational are all major predictors of infant death. Hill [2] conducted another study in Kenya that found that the mother's level of education influences child mortality. Literate women, according to these researches, are aware of infection and disease prevention methods such as use of mosquito nets and immunizations. They are more likely to take sick children to the hospital as soon as possible and adhere to the physicians' instructions. These mothers are aware of germ theory and prioritize sanitary measures in the households.

Many studies have found that the size of a family impacts child mortality rates. When a large group of people stay together, the danger of contracting diseases increases, and hygiene may deteriorate, according to Woldemicael et al. [15]. Due to congestion and competition for resources and the mother's attention, a home with many children is more likely to have disease infections [15].

\subsection{Environmental Factors}

Our overall health is influenced by the environment we live in. Toilet facilities, sources of drinking water, and waste disposal methods are some of environmental factors that affect child mortality.

The health benefits of drinking safe and clean water have been documented in several studies. Analyzing demographic health survey data from 8 sub-Saharan African countries, Fayehun [18] found that countries with lower child mortality rates, such as Lesotho and Namibia, have more households with improved sources of water than those with poor sources of drinking water. Despite having access to water from safe and upgraded sources, Mutunga [16] claims that fetching it in dirty containers and storing it improperly can contaminate it with infection-causing organisms.

According to studies, children who live in homes with improved toilet facilities are less likely to become ill than children who live in homes with no or inadequate toilet facilities. According to a research conducted in Ghana, the risk of catching infections is significantly linked to the availability of toilet facilities, with children living in houses with toilets being around $50 \%$ less likely to contract diseases than those living in houses without toilets [19].

\section{Concepts of Survival Models}

The study of time-to-event occurrences is known as survival analysis. The time to event, also known as survival time, event time or failure time, is a variable that can be quantified in days, weeks, months, or years.

Methods of survival analysis look at data with three key characteristics:

i. The response or dependent variable is the waiting time until a well-defined event occurs.

ii. The observations are censored.

iii. There are predictors that we wish to assess or control the effect on the waiting time.

\subsection{Survival Analysis Data Structure}

There are no definite starting and finishing points in survival analysis. All of the observations do not begin at zero. A subject can join the study at any time. All of the subjects are brought to the same starting point, with the time $t$ set to zero. At time zero, all subjects' survival probabilities are one, meaning they have a 100 percent chance of not experiencing the event of interest, such as death.

\subsection{Censoring}

Censoring refers to a situation where the event of interest fails to be observed for some individuals during the study period. This is what makes survival analysis methods different from other regression methods of analysis. Censoring can take three different forms:

a) A subject has not yet experienced the event of interest at the end of the study.

b) A subject has been lost to follow-up during the study period.

c) A subject has experienced a different event other than the event of interest, making follow-up impossible.

\subsection{Important Functions in Survival Analysis}

The first step in conducting the analysis of survival data is estimating the distribution of survival times.

\subsubsection{Survival Function, $S(t)$}

The survivor function, also known as the survival distribution time, describes the population's survival experiences.

Let $(T \geq 0)$ denote a positive random variable that represents the time to the event, and $F(t)=\operatorname{Pr}(T \leq t)$ denote a Lifetime Distribution Function, which is the 
probability that an event of interest will occur before time $t$. The survival experience of subjects under study is described by the survival function, $S(t)$. by:

Given the time to event $T$, survival function, $S(t)$ is given

$$
S(t)=\operatorname{Pr}(T>t)=1-F(t)
$$

It expresses the likelihood of an individual surviving from the time of origin to a future time $t$. The survival function is a non-increasing function of $t$ that approaches 0 as time advances to infinity, and it is between zero and one (inclusive).

\subsubsection{The Hazard Function ( $\lambda$ )}

The hazard function, also known as the instantaneous failure rate, instantaneous death rate, or force of mortality, is one of the ways for modeling data distribution in survival analysis.

The probability that a subject will succumb to the event in the next instant if it survives to time $t$ is given by the hazard function. In other words, it is the likelihood that an individual will experience the event of interest within a short period of time, as long as the individual survives until the start of that interval. It is given by:

$$
\lambda(t)=\lim _{\Delta t \rightarrow 0} \frac{\{P(t \leq T<t+\Delta t / T \geq t)}{\Delta t}
$$

NOTE: Unlike the survival function, which is concerned with not experiencing the event, the hazard function is concerned with the event occurring.

\subsubsection{The Cumulative Hazard Function, $\Lambda(t)$}

This is a function that describes the accumulated risk up to time $t$.

$$
\Lambda(t)=\int_{0}^{t} \lambda(u) d u
$$

\subsection{Distribution-Free Survival Methods}

The survival and hazard functions are used to summarize the data for non-parametric survival data estimation. The goal of utilizing non-parametric approaches to evaluate survival data is to produce graphical summaries of survival times for a specific group of study subjects. These are the graphical summaries for survival and hazard functions. The median and other percentiles can also be obtained for a more detailed analysis.

\subsubsection{The Empirical Survival Function}

The empirical survival function can be used to estimate the survival function $S(t)$, assuming that all observations are independent and that no data point is censored. In the absence of censored data, the empirical function estimates the survival function.

It is given by:

$$
\widetilde{S}(\mathrm{t})=\frac{\text { Number of individuals with } \mathrm{T}>\mathrm{t}}{\text { Total sample size }}
$$

$\tilde{S}(t)=1$ for all values of $\mathrm{t}$ before the first failure and $\widetilde{S}(t)=0$ after the final occurrence of the event of interest.
Note that $F_{n}(t)=1-S_{n}(t)$ is the empirical cumulative distribution function.

\subsubsection{The Kaplan-Meier Estimator}

The Kaplan-Meier model is a popular non-parametric survival function estimator. The model can be used to generate univariate descriptive statistics for survival data and to compare survival experiences across groups of study subjects. For censored observations, the Kaplan-Meier estimator, also known as the product limit estimator, provides an estimate of the survival function.

The Kaplan-Meier estimator of the survivorship function $S(t)=P(T>t)$ is given by:

$$
\begin{gathered}
\tilde{S}(t)=\prod_{i=1}^{k} \frac{n_{i}-d_{i}}{n_{i}} \\
=\prod_{i=1}^{k}\left(1-\frac{d_{i}}{n_{i}}\right)
\end{gathered}
$$

where:

$i$ is a set of $\mathrm{K}$ distinct uncensored failure times observed in the sample.

$d_{i}$ is the number of failures at $i$

$n_{i}$ is the number of individuals "at risk" right before the $i^{\text {th }}$ failure time (everyone who died or censored at or after time $i$ )

The standard error is therefore given by:

$$
\text { s. e } \tilde{S}(t)=\tilde{S}(\mathrm{t})\left[\sum_{i=1}^{k} \frac{d_{i}}{n_{i}\left(n_{i}-d_{i}\right)}\right]^{\frac{1}{2}}
$$

\subsubsection{Cumulative Hazard Function}

Given $\widetilde{S}(\mathrm{t})$ as the Kaplan-Meier estimator for the survival function, then the cumulative hazard function estimator is given by:

$$
\widetilde{\Lambda}(t)=-\sum_{\mathrm{i}=1}^{\mathrm{k}} \ln \left(1-\frac{\mathrm{d}_{\mathrm{i}}}{\mathrm{n}_{\mathrm{i}}}\right)
$$

Using Taylor series of expansion, we get:

$$
\ln \left(1-\frac{d_{i}}{n_{i}}\right)=-\frac{d_{i}}{n_{i}}-\left(\frac{d_{i}}{n_{i}}\right)^{2}+\cdots,-\left(\frac{d_{i}}{n_{i}}\right)^{k}
$$

Ignoring higher order terms, the cumulative hazard function becomes:

$$
\widetilde{\Lambda}(\mathrm{t})=\sum_{\mathrm{i}=1}^{\mathrm{k}} \frac{\mathrm{d}_{\mathrm{i}}}{\mathrm{n}_{\mathrm{i}}}
$$

Furthermore, in case of censoring:

$$
n_{i}=n_{i-1}-d_{i-1}-c_{i-1}
$$

where, $c_{i}$ is the number of censored observations.

Note that:

a) $\tilde{S}(t)$ only changes at failure times

b) $\tilde{S}(t)$ is until the first failure time

c) $\tilde{S}(t)$ only goes to 0 if the last observation is not censored

The Kaplan-Meier estimator equals the empirical survival estimator when there is no censoring. 


\subsection{Parametric Survival Methods}

The survival times in these models are assumed to follow a specified probability distribution. Weibull, Exponential, Log-logistic and Gama are the most commonly used distributions.

\subsubsection{Exponential Distribution}

The hazard rate in the exponential model is constant hazard $\lambda(t)=\lambda$.

In other words, the probability that a subject will experience the event of interest within a short time interval given that it has not experienced the event at the start of the interval is constant for any time period. Therefore, the survival function $S(t)$, which is the probability of surviving beyond time $t$, is:
And the density is:

$$
f(t)=\lambda \exp (-\lambda t)
$$

The hazard function $\lambda(t)$ can be obtained from both the density and survival as follows:

$$
\lambda(t)=\frac{f(t)}{S(t)}=\lambda
$$

Finally, the cumulative hazard function is given by:

$$
\Lambda(t)=\lambda t
$$

The constant hazard rate gives the exponential distribution has a unique property known as the loss of memory property.

$$
S(t)=\exp (-\lambda t)
$$

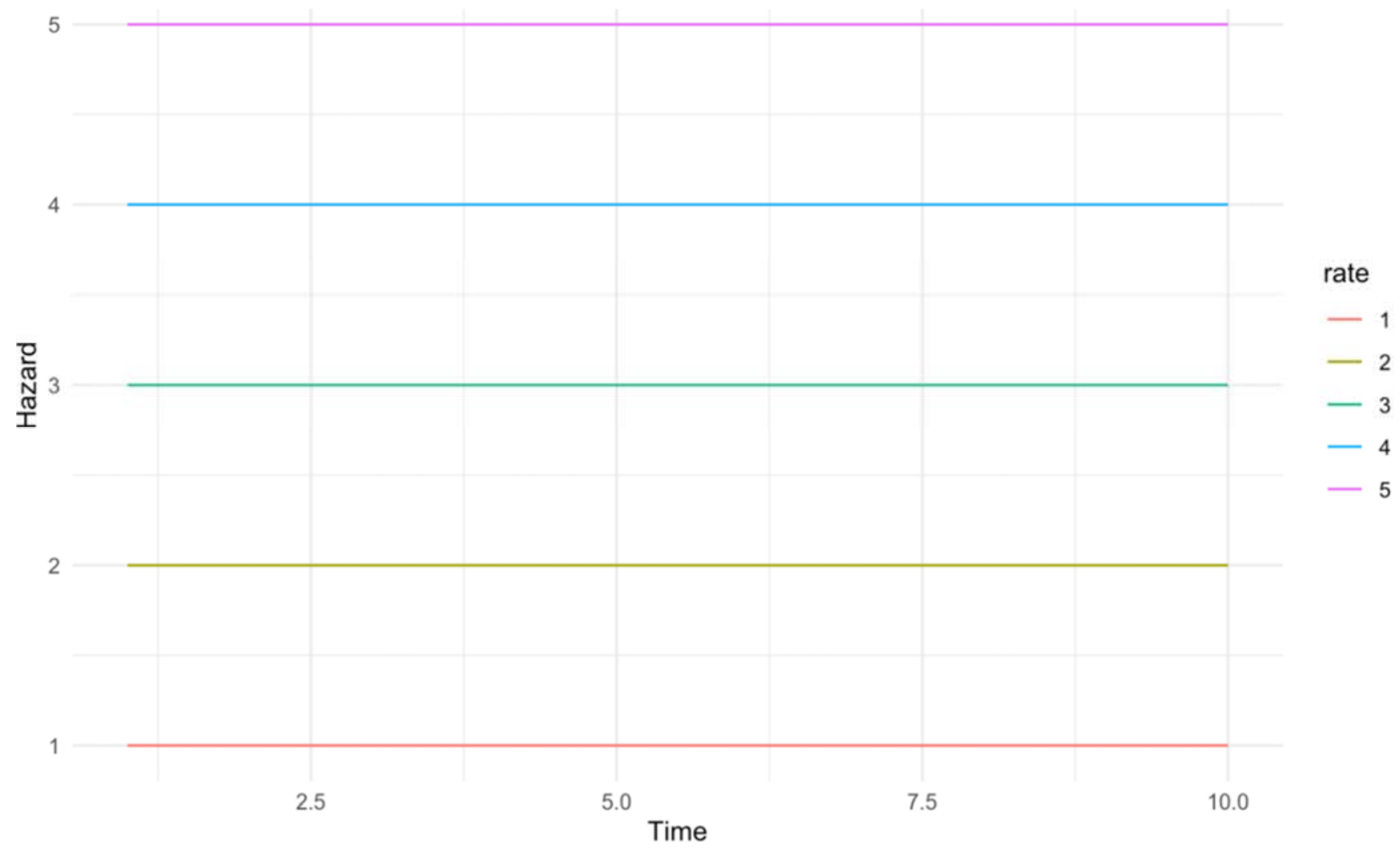

Figure 1. The shapes of the Exponential hazard functions for some selected values of the shape parameter.

\subsubsection{Weibull Distribution}

The Weibull distribution is a flexible model with two parameters; the scale, $\lambda$, and shape, $\kappa$, parameters. Its flexibility makes it a perfect model that can be used to model survival times of populations whose hazard rate is decreasing $(\kappa<1)$, increasing $(\kappa>1)$, or constant $(\kappa=1)$. The Weibull distribution simplifies to the Exponential distribution when $\kappa=1$.

The cumulative density function $F(t)$ is given by:

$$
F(t)=1-\exp (-[t \lambda] \alpha)
$$

The survival function denoted by $S(t)$ is given as:

$$
S(t)=\exp \left(-\lambda t^{\kappa}\right)
$$

The density function $f(t)$ is given by:

$$
f(t)=\frac{-d}{d t} S(t)=\kappa \lambda t^{\kappa-1} \exp \left(-\lambda t^{\kappa}\right)
$$

The hazard rate is given by:

$$
\lambda(t)=\kappa \lambda t^{\kappa-1}
$$

Therefore, the cumulative hazard function for the Weibull hazard distribution is given by: 


$$
\lambda(t)=\int_{0}^{t} \lambda(u) d u
$$
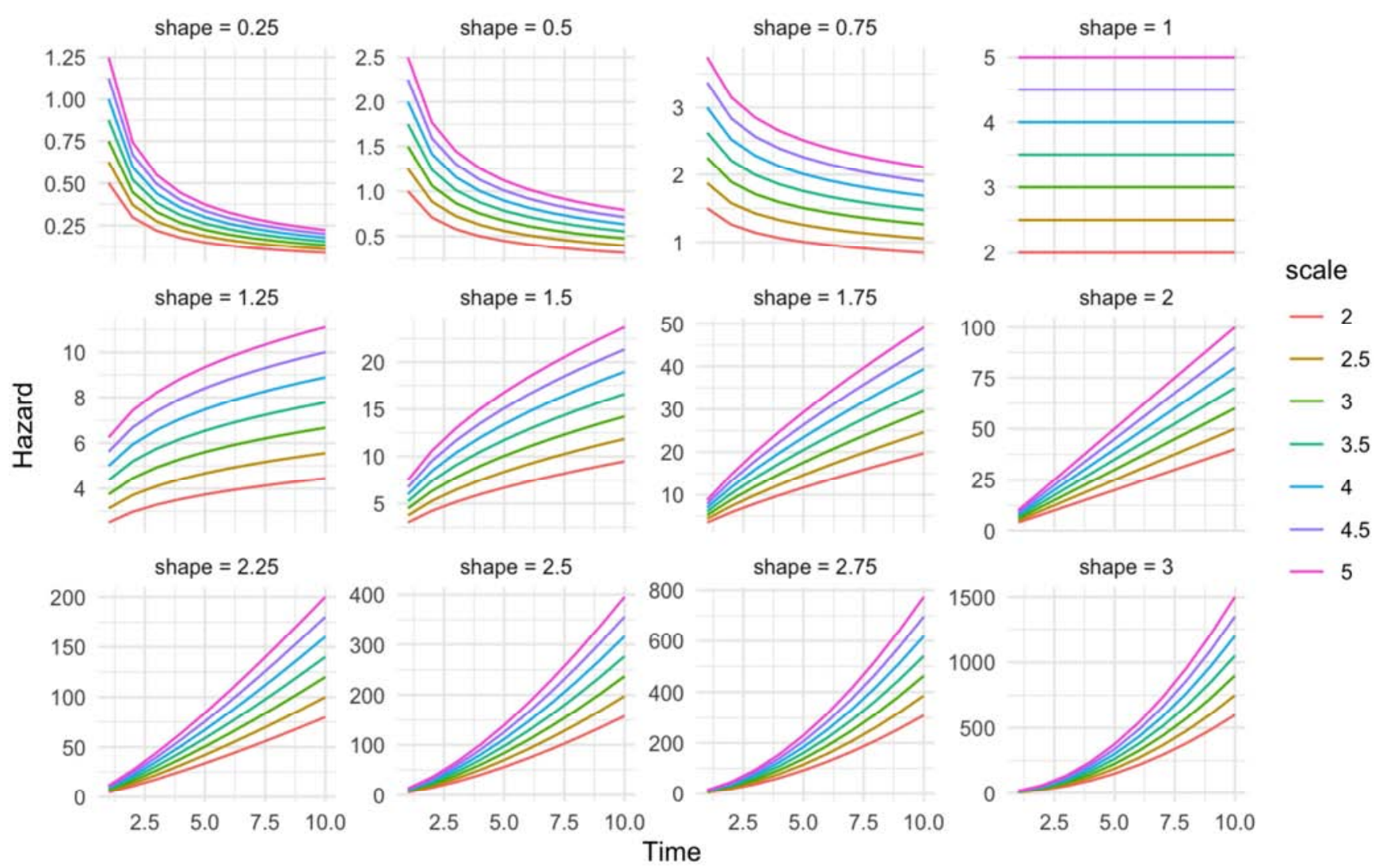

Figure 2. The shapes of the Weibull hazard functions for some selected values of the shape parameter.

\subsubsection{Log-normal Distribution}

The Log-normal model, like the Weibull distribution, is a fairly flexible distribution, with the ability to fit many types of failure times data. It has scale, $\delta$, and shape, $\tau$ parameters.

If $T$ has log-normal distribution, then:

$$
Y=\log (T)=\delta+\tau W
$$

Where $\mathrm{W}$ is the standard logistic distribution with a density given by:

$$
f_{\tau}(\tau)=\frac{e^{\tau}}{\left(1+e^{\tau}\right)^{2}}
$$

The $\mathrm{CDF}$ is given by:

$$
F_{\tau}(\tau)=\frac{e^{\tau}}{\left(1+e^{\tau}\right)}
$$
by:

The survival function is the complement of the CDF given

$$
S_{\tau}(\tau)=\frac{1}{\left(1+e^{\tau}\right)}
$$

Therefore, for the variable $T$, the survival function is given by:

$$
S_{\tau}(\tau)=\frac{1}{(1+\lambda t)^{p}}
$$

where $p=\frac{1}{\delta}$ and $\delta=-\log \lambda$

When the observations follow a log-normal distribution, the $\log$ of the observations follows a normal distribution with mean $L n \tau m$ and standard deviation $\delta$. This property gives the log-normal distribution flexibility as the failure times can be analyzed as normally distributed data.

\subsection{Regression Models in Survival Analysis}

The statistical goal of survival analysis, just like linear and logistic regression, is to describe the relationship between time to failure and some predictor variables. The link between survival time and a set of covariates is examined using parametric, semi-parametric, and non-parametric survival models. 


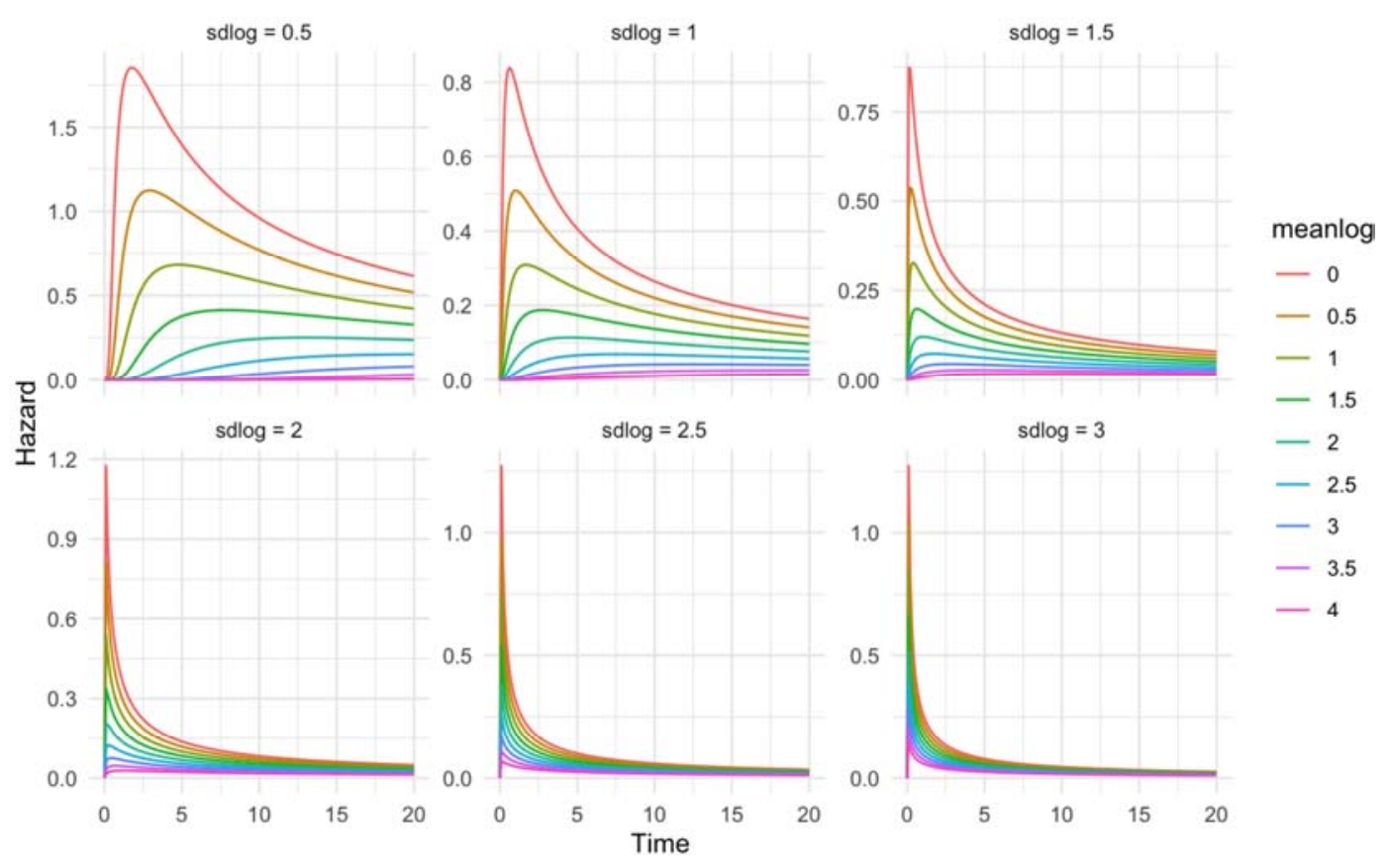

Figure 3. The shapes of the Log-normal hazard functions for some selected values of the shape parameter.

\subsection{Regression Models in Survival Analysis}

The statistical goal of survival analysis, just like linear and logistic regression, is to describe the relationship between time to failure and some predictor variables. The link between survival time and a set of covariates is examined using parametric, semi-parametric, and non-parametric survival models.

\subsubsection{Parametric Regression Models}

The Weibull and exponential distributions are the most commonly used parametric survival regression models. Parameter estimates in parametric survival models are obtained using maximum likelihood estimation, just like in logistic regression. Thus, in parametric survival analysis, we can apply the same procedures for testing and generating confidence intervals as we do in logistic regression.

Exponential Regression Model

The survival function of $T$ at covariate values $X=$ $\left(x_{1}, x_{2}, \ldots . x_{n}\right)^{T}$ can be shown to be:

$$
S(t \mid x)=\exp \left[-\left(t e^{-} X^{T} \beta\right)^{\frac{1}{\delta}}\right)
$$

where $\beta=\left(\beta_{0}, \beta_{1}, \ldots, \beta_{n}\right)^{T}$ is a vector of regression coefficients.

The hazard function is given by:

$$
\lambda(t \mid x)=\lambda \exp \left(X^{\prime} \beta\right)
$$

In log terms, the log hazard function is given by:

$$
\log \lambda(t \mid X)=\left(\frac{1}{\delta}-1\right) \log t-\log \delta-X^{T}\left(\frac{\beta}{\delta}\right)
$$

The model transforms into a linear model for $Y=\ln (T)$.

Weibull Regression Model

The hazard function is normally given by:

$$
h(t)=\delta \lambda^{\delta} t^{\delta-1}
$$

To include the covariate vector $\mathrm{X}$, we write the hazard given $\mathrm{X}$ as:

$$
\begin{gathered}
\lambda(t \mid X)=\lambda_{0}(t) \cdot \exp \left(X^{\prime} \beta\right) \\
=\delta \lambda^{\delta} t^{\delta-1} \cdot \exp \left(X^{\prime} \beta\right) \\
=\delta \lambda^{\delta} t^{\delta-1}
\end{gathered}
$$

where $\lambda=\lambda \cdot\left(\exp \left(X^{\prime} \beta\right)\right)^{\frac{1}{\delta}}$

Just like the exponential model, we get the following result in log-scale:

$$
\begin{gathered}
\ln (\lambda(t \mid x))=\ln (\delta)+\delta \ln (\lambda)+(\delta-1) \\
\ln (t)=\ln (\delta)+\delta \ln (\lambda)+X^{\prime} \beta+(\delta-1) \ln (t)
\end{gathered}
$$

\subsubsection{Non-parametric Regression Model: The Cox Proportional Hazard Model}

A popular semi-parametric method for assessing survival data is the Cox proportional hazard model (Cox PH). The model uses the hazard function to explore the association between time-to-event and a set of predictors. The Cox model is based on the premise that the predictors have an increasing effect on the hazard function and that the effect is constant over time.

The hazard function is given by:

$$
\begin{gathered}
\lambda(t \mid x)=h_{0}(t) e^{X^{\prime} \beta} \\
=\lambda_{0}(t) e^{X^{\prime} \beta_{1} x_{1}+}
\end{gathered}
$$

where

$\lambda_{0}(t)$ is the baseline hazard, which does not depend on the covariates, 
$\lambda(t \mid x)$ is the hazard at time $t$ for an individual with a set of predictor variables $X$,

$\beta$ is a set of parameters that describe the effect of the covariates on the overall hazard.

Thus, we interpret the Cox hazard model using hazard ratios, defined as the ratio of estimated hazard function of two values of covariates. The event of interest is less likely to occur when the hazard ratio is less than 1 , and it is more likely to occur when the hazard ratio is more than 1 . When the hazard ratio equals 1 , the predictor variable has no effect on the event's hazard function.

Thanks to its regression framework, the Cox model has an advantage of allowing us to estimate hazard ratios that are controlled for other factors in the model, such as race, sex, and age. Another advantage of the Cox model is that they do not require one to assume a specific survival distribution for the data. Instead, the models use a hazard function.

\subsubsection{Proportional Hazard Property}

Despite its apparent simplicity, the Cox model has a key assumption that must be checked before it can be used. Regardless of how the underlying hazard function changes over time, the Cox model assumes proportionate hazards between predictor values.

In other words, the hazard ratio for two different observations, $x_{1}$ and $x_{2}$, the hazard ratio is as follows:

$$
\begin{aligned}
& \frac{h\left(t \mid x_{1}\right)}{h\left(t \mid / x_{2}\right)}=\frac{\exp \left(x_{1}^{\prime} \beta\right)}{\exp \left(x_{2}^{\prime}{ }_{2} \beta\right)} \\
= & \exp \left(\left(x_{1}^{\prime}{ }_{1}-x_{2}^{\prime}\right) \beta\right)
\end{aligned}
$$

This implies that the hazard ratio of the two observations is constant with respect to time $t$.

For any Cox proportional hazard model, the survival function for $T$ is as follows:

$$
\begin{gathered}
S(t \mid x)=\exp \left(\int_{0}^{t} \lambda(u \mid x) d u\right) \\
=\exp \left(-\exp \left(X^{\prime} \beta\right) \int_{0}^{t} \lambda_{0}(u) d u\right) \\
=\exp \left(\int_{0}^{t} \lambda_{0}(u) d u\right)^{\exp \left(X^{\prime} \beta\right)}=\left(S_{0}(t)\right)^{\exp \left(X^{\prime} \beta\right)}
\end{gathered}
$$

where $S_{0}(t)$ is the baseline hazard function.

Therefore, the probability distribution function of $T$ is given by:

$$
f(t \mid x)=\lambda(t \mid x) S(t \mid x)=\lambda_{0}(t) \exp \left(X^{\prime} \beta\right)\left(S_{0}(t)\right)^{\exp \left(X^{\prime} \beta\right)}
$$

Note that if the proportionality assumption holds, then

$$
S_{0}(t)=\left(S_{0}(t)\right)^{\exp \left(X^{\prime} \beta\right)}
$$

Because the model is based only on this assumption, if it proves to be false for a number of covariates in a given data set, the model should not be applied to that data set. Fitting a model with time-varying covariates or a stratified Cox model where the baseline hazard varies from stratum to stratum is one technique to get around this challenge.
In a Cox model, it's important to check the proportional hazard assumption for each predictor. Plotting the Schoenfeld residuals versus time is the best way to achieve this (for continuous variables). For a valid proportional hazard assumption, the Schoenfeld residuals should resemble a random scatter around 0 .

For categorical variables, compare log-log transformation of the Kaplan Meier survival curves for different categories. For a valid proportional hazard assumption, the survival curves will be almost parallel and will intersect after time apart. There might be a bit of crossing at earlier time points caused by noise in the estimates, which may violate the assumption.

\section{Application to Application to the 2014 Kenya Demographic Health Survey Data}

\subsection{Introduction to Data}

The data for this study came from the Kenya Demographic and Health Survey of 2014 (KDHS 2014). The DHS is a nationwide representative survey of men and women aged 15 to 54 and 15 to 49 , respectively, drawn from 5,360 clusters around the country. All the sampled households received the Women's Questionnaire and the Household Questionnaire, whereas every second sampled household received the Men's Questionnaire.

Just like with any other child mortality data, important information for this study comes from surveys conducted on women. The interviews entailed a retrospective maternal history, which included information on each child's birth date, survival status, and death age.

The outcome variable in the study is the duration of time from when a child is born to death or censor. The main covariates are categorized into environmental (drinking water and type of toilet facility), socioeconomic (place of residence, family wealth index, place of delivery, and mothers education), and demographic (mother's age at birth, sex of a child, family size, breastfeeding habits, and preceding interval) factors.

\subsection{Descriptive Analysis}

Out of the 83,591 children who were born alive between 2008/9 and 2014, 50.6\% were males while 49.4\% were females. Over two-thirds $(69.8 \%)$ of the children were born in rural areas, while only $30.2 \%$ were born in urban areas of Kenya. Nairobi region had the least number of children born (1.9\%) between 2008/9 and 2014. According to the findings, the poorest households had the highest proportion of children $(31.3 \%)$ compared to the richest households (12.3\%). Slightly more than three-quarters $(75.7 \%)$ of the children born alive lived with their mothers, $7.6 \%$ lived with relatives, and only $6.7 \%$ lived with their fathers.

Women who had the lowest level of education (primary or no education at all) gave birth to most children compared to their learned counterparts (secondary and higher education), who gave birth to only $21.7 \%$ of the total number of children 
born alive. Almost all the children born between 2008/9 and 2014 came from Christian households (81.4\%), and only $15.8 \%$ came from Muslim households. A higher proportion of children were born to self-employed mothers (83\%), with only $3.9 \%$ being born to employed mothers.

Male-headed households accounted for $64.1 \%$ of the children born between 2008/9 and 2014, while female-headed households accounted for $35.9 \%$.

According to the findings, out of the 83,591 children born alive between 2009 and 2014, 6,123 (7.3\%) did not celebrate their fifth birthday. A higher proportion of the deaths occurred among male children (54.6\%) than among female children (45.4\%).

A child born in the Nyanza region (136 deaths per 1,000 live births) is twice as likely as a child born in the Central region ( 58 deaths per 1,000 live births) to die before reaching the age of five. The Western region had the second-highest number of deaths among children under the age of five (115 deaths per 1,000 live births). The majority of under-five deaths $(70.9 \%)$ happened in rural areas, with only $29.1 \%$ occurring in Kenya's urban areas.

As expected, the majority of children who died before reaching the age of five came from the poorest families $(32.6 \%)$, with only $8.4 \%$ from the wealthiest families. When compared to other birth orders, firstborn children had the highest rate of deaths $(28.2 \%)$. Children whose mothers had a lower level of education (primary and below) died the most $(84.9 \%)$, while only $2.4 \%$ occurred to children whose mothers had a higher level of education.

The majority of the children who died before five years came from male-headed households $(62.8 \%)$ than from female-headed households $(37.2 \%)$. The results indicate that children born as a result of multiple births recorded the highest number of deaths than those born as a result of singleton births. Of the 2171 multiple births, 450 (20.7\%) died before reaching the age of five, while only $6.2 \%$ of singleton births died before the age of five. Table 1 summarizes the distribution of live births and deaths among children under the age of five.

Table 1. Distribution of births and deaths by survival determinants.

\begin{tabular}{|c|c|c|c|c|c|}
\hline Covariates & & Frequency & Proportion & Child is Dead & Child is Alive \\
\hline \multirow{2}{*}{ Sex of Child } & Male & 42,337 & 50.6 & 54.6 & 50.3 \\
\hline & Female & 41,254 & 49.4 & 45.4 & 49.7 \\
\hline \multirow{2}{*}{ Place or Residence } & Urban & 25,261 & 30.2 & 29.1 & 30.3 \\
\hline & Rural & 58,330 & 69.8 & 71 & 69.7 \\
\hline \multirow{8}{*}{ Region } & Coast & 10,350 & 12.4 & 14.1 & 12.2 \\
\hline & North Eastern & 5,738 & 6.9 & 5.9 & 6.9 \\
\hline & Eastern & 13,109 & 15.7 & 12 & 16 \\
\hline & Central & 6,678 & 8 & 6 & 8.1 \\
\hline & Rift Valley & 25,367 & 30.3 & 21.8 & 31 \\
\hline & Western & 8,145 & 9.7 & 13.7 & 9.4 \\
\hline & Nyanza & 12,635 & 15.1 & 24.7 & 14.4 \\
\hline & Nairobi & 1,569 & 1.9 & 1.6 & 1.9 \\
\hline \multirow{3}{*}{ Mother's Educational Level } & No Education & 18,303 & 21.7 & 21.9 & 21.9 \\
\hline & Primary & 47,126 & 56.4 & 63 & 55.9 \\
\hline & Higher & 3,942 & 4.7 & 2.4 & 4.9 \\
\hline \multirow{5}{*}{ Wealth Index } & Poorest & 26,170 & 31.3 & 32.6 & 31.2 \\
\hline & Poorer & 17,926 & 21.4 & 25.2 & 21.1 \\
\hline & Middle & 15,905 & 19 & 19.4 & 19 \\
\hline & Richer & 13,316 & 15.9 & 14.3 & 16.1 \\
\hline & Richest & 10,271 & 12.3 & 8.4 & 12.6 \\
\hline \multirow{2}{*}{ Sex of HH Head } & Male & 53,109 & 64.1 & 62.8 & 64.2 \\
\hline & Female & 29794 & 35.9 & 37.2 & 35.8 \\
\hline \multirow{4}{*}{ Religion } & Roman Catholic & 15,859 & 19 & 16.7 & 19.2 \\
\hline & Protestants & 52,214 & 62.5 & 64.4 & 62.3 \\
\hline & Muslims & 13,163 & 15.7 & 16.1 & 15.7 \\
\hline & Other Religion & 2,355 & 2.8 & 2.8 & 2.8 \\
\hline \multirow{3}{*}{ Mother's Age } & $15-19$ & 1,080 & 1.3 & 0.5 & 1.4 \\
\hline & $20-29$ & 21,210 & 25.4 & 18.3 & 25.9 \\
\hline & $30-39$ & 33,026 & 39.5 & 36.6 & 39.7 \\
\hline \multirow[t]{2}{*}{ Mother's Occupation } & Employed & 3,227 & 3.9 & 3.7 & 3.9 \\
\hline & Self Employed & 69,379 & 83 & 84.7 & 82.9 \\
\hline \multirow{5}{*}{ Birth Order } & 1st Birth & 23,245 & 27.8 & 28.2 & 27.8 \\
\hline & 2nd Birth & 18,819 & 22.5 & 21.7 & 22.6 \\
\hline & 3rd Birth & 14,081 & 16.8 & 15.9 & 16.9 \\
\hline & 4th Birth & 9,963 & 11.9 & 11.7 & 11.9 \\
\hline & 5th \& Higher Births & 17,483 & 20.9 & 22.6 & 20.8 \\
\hline
\end{tabular}




\subsection{Non-parametric Survival Analysis: Kaplan-Meier Model}

The Kaplan-Meier curves for a few selected covariates for under-five child mortality in Kenya are shown in the figures below.

As shown in the graphs below, a male child has a higher risk of dying before reaching the age of five than a female child. Figures also show that the Rift Valley and Central regions have lower death rates than the rest of the country. The Western and Nyanza regions, on the other hand, have high rates of under-five mortality. This means that children born in the Western or Nyanza regions are more likely than those born in the Central to die before the age of five.

Figures 4 show that a child born to a mother with
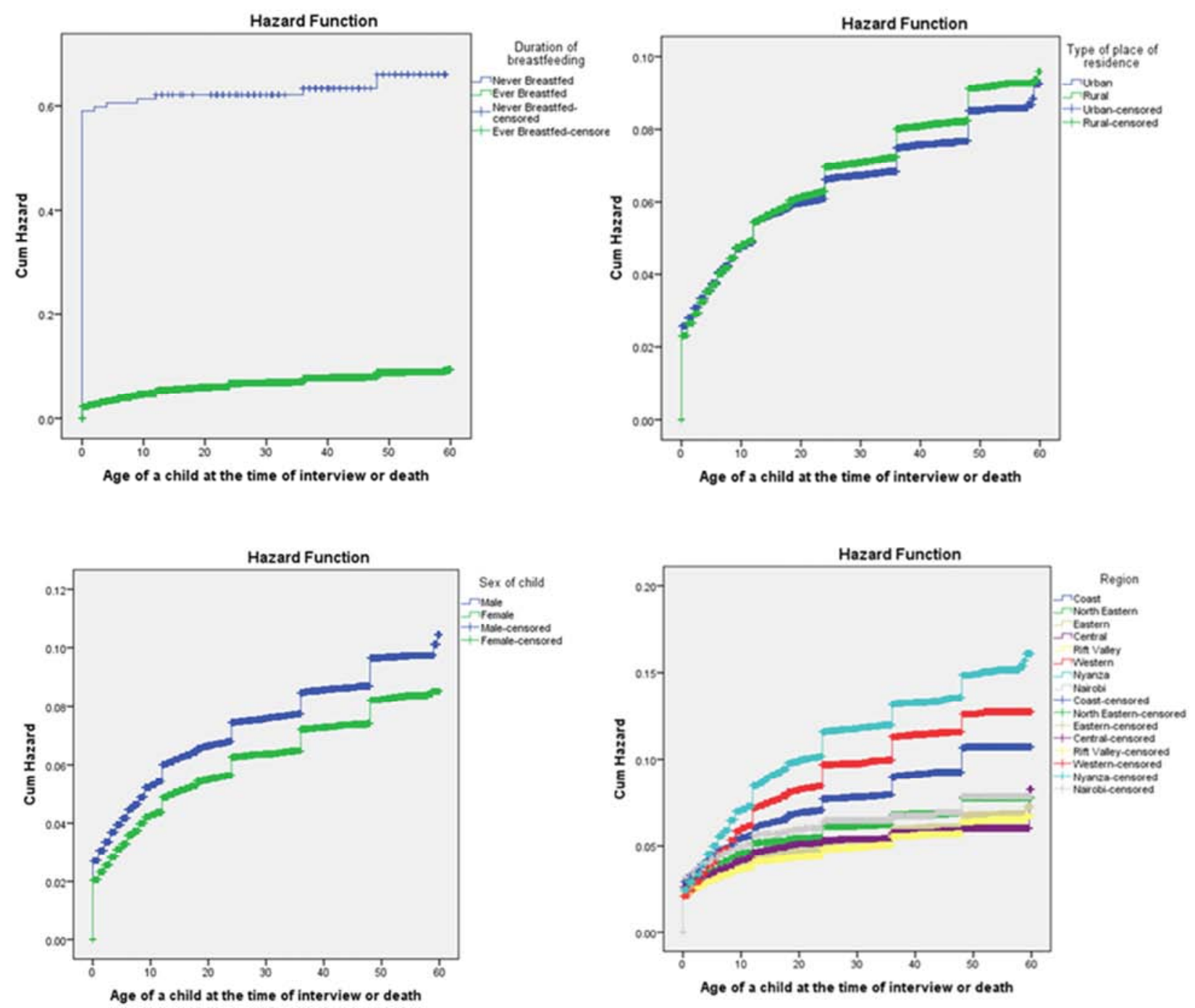

Figures 4. The Kaplan-Meier curves for a few selected risk factors for under-five child mortality in Kenya from KDHS 2014.

\subsection{Fitting a Standard Cox-proportional Hazard Model on the KDHS 2014 Data}

The Cox PH model with several independent variables was fitted with an assumption that the times to death of all children secondary or higher level of education has a better chance of surviving beyond the age of five than a child born to a mother with primary or lower level of education. The survival curve for children born in urban area is higher than the curve for children born in rural areas. In other words, children born in urban have a better chance of surviving until they reach the age of five than children born in rural areas.

A child's survival is also dependent on his or her family's wealth. A child born into the richest family has a better chance of surviving than a child born into a poorer household, as indicated in the figures below. Breastfed children experienced a lower risk of dying before attaining five years than those who were not. 
predictor of under-five child mortality. Attending secondary education and above is strongly linked to a lower risk of child mortality. A child born to a mother with a higher level of education had a better probability of surviving to the age of five $(b=-0.063 ; S E=0.087 ; p-$ value $<0.01)$ than a child born to a mother with a secondary $(b=-0.026$; $S E=$ $0.045 ; p$-value $<0.01)$ or lower-level of education ( $b=0.113 ; S E=0.032 ; p-$ value $<0.01)$.

The region where a child is born and raised has an impact on his or her odds of surviving to the age of five. The significant positive coefficients for both the Western $(b=$ $0.187, S E=0.048, p-$ value $<0.01)$ and Nyanza $(b=$ $0.352, S E=0.043, p-$ value $<0.01)$ regions indicate that the risk of under-five mortality is higher in these two regions than in other parts of the country. This means that children born in the Nyanza and Western are more likely than those born in other regions to die before reaching the age of five.
Being born in the Central, Nairobi, Rift Valley, and North Eastern regions, on the other hand, was found to be negatively associated with the risk of under-five mortality (Central: $\$ b=-0.428, S E=0.062, p-$ value $<0.01 \quad \$ ; \quad$ Nairobi: $b=-0.232, S E=0.105, p-$ value $=0.01$; Rift Valley: $b=-0.466, S E=0.044, p-$ value $<0.01$; North Eastern: $b=-0.266, S E=0.062, p-$ value $<0.0)$. In other words, children born in these areas experience lower mortality rates than those born in the Western and Nyanza parts of the country.

There is a strong positive correlation between place of residence $\quad(b=0.061, S E=0.033, p-$ value $=0.013)$ and the survival of under-five children. Children born in rural areas face a higher risk of under-five mortality than children born in urban areas. Simply put, children born in Kenya's rural areas have a higher likelihood of dying before reaching the age of five than children born in the country's urban settings.

Table 2. The Univariate Cox Proportional Hazard Model.

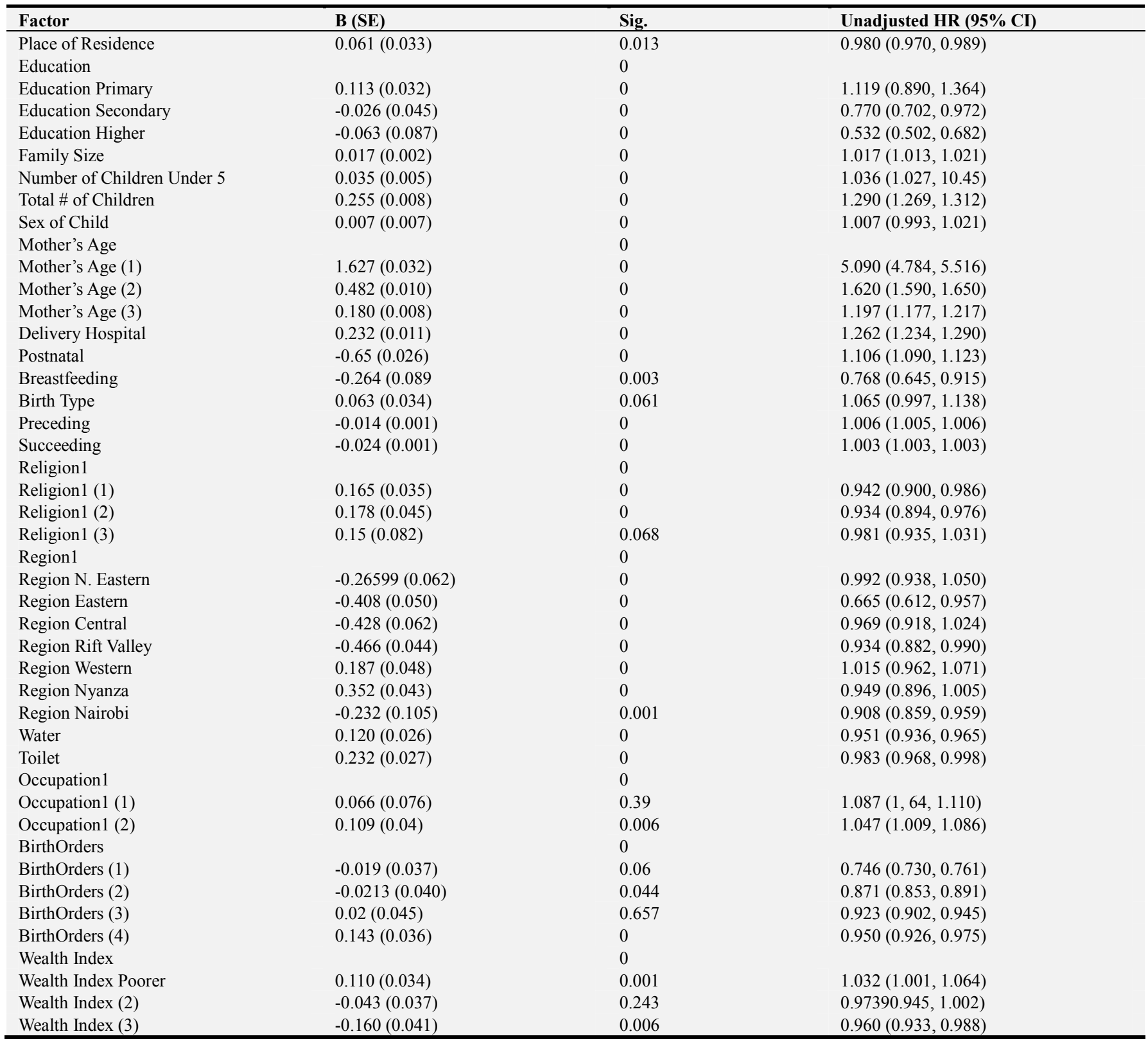




\begin{tabular}{llll}
\hline Factor & B (SE) & Sig. & Unadjusted HR (95\% CI) \\
\hline Wealth Index (4) & $-0.421(0.05)$ & 0.072 & $0.975(0.948,1.002)$ \\
Sex of HH Head & $0.042(0.078)$ & 0.596 & $1.042(0.894,1.216)$ \\
\hline
\end{tabular}

The significant positive coefficient $(b=0232, S E=$ 0.027), $p$-value $<0.01)$ indicates that the risk of under-five mortality is higher in children who live in households with unimproved toilet facilities than in children who reside in households with improved toilet facilities.

The source of a household's drinking water has a strong link to under-five child mortality. Children born in households with unsafe sources of drinking water have a higher chance of dying before reaching the age of five than children born in households with safe sources of drinking water $(b=$ 0.120; $S E=0.026 ; p-$ value $<0.01)$.

The death rate of children under the age of five rises with the size of a family $(b=0.017, S E=0.002, p-$ value $<$ 0.01). In other words, children born into households with more family members are more likely to die before reaching the age of five than children born into smaller families.

The total number of children under the age of five in a home was found to be a significant positive predictor $(b=$ $0.105, S E=0.005, p-$ value $<0.01) \quad$ of under-five mortality. This means that a child born into a family with more under-five-year-old children is more likely to die before reaching the age of five than a child born into a family with fewer under-five-year-old children. The number of total children ever born was also found to be a significant negative predictor of under-five mortality $(b=0.255, S E=$ $0.008, p-$ value $<0.01)$. This indicates that as the number of children born in a family grows, so does the likelihood of a child born in that household dying before reaching the age of five.

The preceding birth interval was a significant negative predictor of under-five mortality $(b=-0.014, S E=0.001$, p-value 0.01). In other words, increasing the length of the preceding birth interval increases the likelihood of children surviving beyond five. The higher succeeding birth interval was found to be a significant negative predictor $(b=$ $-0.024 ; S E=0.001 ; p-$ value $<0.01$ ) of the hazard of under-five mortality. Simply put, increasing the length of succeeding birth interval reduces the risk of a child dying before the age of five children. The under-five child mortality rate of twins was higher than that of singletons ( $b=$ 1.291; $S E=0.049 ; p-$ value $=0.061)$.

The occupation of a mother is strongly linked to the survival of children under the age of five. A child born to a working mother $(b=0.109 ; S E=0.04 ; p-$ value $=0.006) \quad$ or self-employed mother $\quad((b=0.066 ; S E=0.079 ; p-$ value $=0.39)$ has a higher likelihood of dying before reaching the age of five than a child born to an unemployed mother.

\subsubsection{Testing Proportional Hazard Assumption Using the Scaled Schoenfeld Residuals}

To perform the multiple covariate Cox PH model, we needed to test for the proportionality hazard assumption. The scaled Schoenfeld residuals test was performed in $\mathrm{R}$ using the cox. zph tool to test for the proportionality hazard assumption. The test's findings are listed in table 3 below.

Table 3. Testing proportional hazard assumption using the scaled Schoenfeld Residuals.

\begin{tabular}{|c|c|c|c|c|c|}
\hline Covariate & Chi-square (df) & p-value & Covariate & Chi-square (df) & p-value \\
\hline Mother's Age & $36.3(1)$ & $<0.01$ & Toilet Facility & $2.84(1)$ & 0.092 \\
\hline GLOBAL & $36.3(1)$ & $<0.01$ & GLOBAL & $2.84(1)$ & 0.092 \\
\hline Family Size & $1.77(1)$ & 0.18 & Religion & $4.82(3)$ & 0.19 \\
\hline GLOBAL & $1.77(1)$ & 0.18 & GLOBAL & $4.82(3)$ & 0.19 \\
\hline Total Children in $\mathrm{HH}$ & $2.33(1)$ & 0.13 & Mother's Occupation & $3.96(2)$ & 0.14 \\
\hline GLOBAL & $2.33(1)$ & 0.13 & GLOBAL & $3.96(2)$ & 0.14 \\
\hline Number of Children Under 5 & $0.573(1)$ & 0.45 & Child is Twin & $17.9(1)$ & $<0.001$ \\
\hline Preceding Birth Interval & $0.518(1)$ & 0.47 & Sex of Child & $7.6(1)$ & 0.005 \\
\hline GLOBAL & $0.518(1)$ & 0.47 & GLOBAL & $7.6(1)$ & 0.005 \\
\hline Succeeding Birth Interval & $137(1)$ & $<0.01$ & Wealth & $15.8(4)$ & 0.0033 \\
\hline GLOBAL & $137(1)$ & $<0.01$ & GLOBAL & $15.8(4)$ & 0.0033 \\
\hline Region & $23.6(7)$ & 0.0013 & Place of Delivery & $35.3(1)$ & $<0.001$ \\
\hline GLOBAL & $23.6(7)$ & 0.0013 & GLOBAL & $35.3(1)$ & $<0.001$ \\
\hline Place of Residence & $2.96(1)$ & 0.086 & Breastfeeding & $37.6(1)$ & $<0.001$ \\
\hline Education & $7.94(3)$ & 0.057 & Postnatal & $6.59(1)$ & 0.01 \\
\hline GLOBAL & $7.94(3)$ & 0.057 & GLOBAL & $6.59(1)$ & 0.01 \\
\hline Source of Water & $0.88(1)$ & 0.35 & Birth Orders & $6.47(4)$ & 0.17 \\
\hline GLOBAL & $0.88(1)$ & 0.35 & GLOBAL & $6.47(4)$ & 0.17 \\
\hline
\end{tabular}

The scaled Schoenfeld residuals test shows that the region, sex of a child, succeeding birth interval, breastfeeding habits, place of delivery, multiple births, wealth index, mother's age at childbirth, and postnatal care do not satisfy the proportionality hazard assumption because their $p$-values are less than 0.05 significance level.

As a result, the adjusted hazard ratios cannot be estimated using the final cox-proportional hazard model. Other factors, such as family size and total number of children in a household, however, meet this requirement and can thus be 
included in the final cox-proportional hazard model.

\subsubsection{The Best Fitting Model Selected by the AIC by Akaike}

Table 4. The best fitting model selected by the AIC by Akaike (1973) of the standard.

\begin{tabular}{|c|c|c|c|c|}
\hline Covariates & B & SE & p-value & HR $(95 \%$ CI $)$ \\
\hline Place of Residence Rural & 0.115 & 0.030 & 0.000 & $1.122(1.057,1.191)$ \\
\hline Education & & & 0.000 & \\
\hline Education Primary & 0.049 & 0.096 & 0.607 & $1.050(0.871,1.267)$ \\
\hline Education Secondary & 0.388 & 0.088 & 0.000 & $1.474(1.239,1753)$ \\
\hline Education Higher & 0.209 & 0.092 & 0.023 & $1.232(1.029,1.476)$ \\
\hline Family Size & -0.220 & 0.007 & 0.000 & $0.803(0.792,0.814)$ \\
\hline Number of Children Under 5 & -0.037 & 0.015 & 0.014 & $0.964(0.936,0.993)$ \\
\hline Preceding & -0.009 & 0.001 & 0.000 & $0.991(0.989,0.993)$ \\
\hline Toilet Unimproved & -0.143 & 0.029 & 0.000 & $0.867(0.819,0.917)$ \\
\hline Religion & & & 0.000 & \\
\hline Religion1 Protestant & -0.033 & 0.083 & 0.688 & $0.967(0.821,1.139)$ \\
\hline Religion1 Muslim & 0.124 & 0.080 & 0.118 & $1.133(0.969,1.324)$ \\
\hline No Religion & 0.271 & 0.083 & 0.001 & $1.311(1.113,1.543)$ \\
\hline Occupation & & & 0.008 & \\
\hline Occupation Employed & -0.060 & 0.041 & 0.146 & $0.942(0.868,1021)$ \\
\hline Birth Order & & & 0.000 & \\
\hline Birth Orders 2nd & 0.603 & 0.041 & 0.000 & $1.827(1.687,1.980)$ \\
\hline Birth Order 3rd & 0.442 & 0.042 & 0.000 & $1.555(1.433,1.688)$ \\
\hline Birth Order 4th & 0.326 & 0.044 & 0.000 & $1.386(1.272,1.510)$ \\
\hline Birth Order 5th and Higher & 0.236 & 0.047 & 0.000 & $1.267(1.155,1.389)$ \\
\hline
\end{tabular}

Using Akaike's Information Criterion (AIC) by Akaike (1973), the best fitting standard Cox proportional hazard model consisted of eleven risk factors, including religion, type of toilet facility, family size, mother's level of education, mother's occupation, place of residence, the total number of children ever born, preceding birth interval, birth order, and number of under- 5 children in a household as illustrated in table 4 above. These results provide enough evidence to conclude that the aforementioned factors have an impact on under-five child mortality in Kenya.

In comparison to those with fewer children and family members, households with a higher number of children as well as total family members had a higher risk of death for children under the age of five. A child born in a rural setting has a greater chance of dying before reaching his or her fifth birthday than a child born in urban areas. An illiterate mother has a greater chance of losing her child before the age of five than a mother who is educated (secondary and above).

\section{Discussion}

The purpose of this research was to find out the risk factors of under-5 mortality in Kenya. According to the findings, 6,123 ( 7.3 percent) of the 83,591 children born alive between 2009 and 2014 did not reach the age of five. Place of residence, preceding birth interval, family size, religion, birth order, mother's occupation, mother's level of education, total number of children ever born, and type of toilet facility were found to be strong predictors of U5CM in Kenya. These findings are similar to those of researches conducted in other Sub-Saharan African countries such Zimbabwe, Ethiopia, Tanzania and
Uganda [13].

A child who was born and raised in an urban setting had a lower risk of dying than a child who was born and raised in a rural area. This finding is consistent with the results of earlier studies [13]. In Kenya, disparities in socioeconomic resources between rural and urban communities, as well as political influence, have a direct impact on health [20]. These findings could also be explained by the fact that urban areas have better sanitation environment and improved health services than rural areas.

Mothers who had more children in the previous five years put their children at risk of dying before they turned five. Short birth intervals are also linked to a high child death rate, according to the findings. These findings could be explained by maternal depletion syndrome, resource competition among siblings, and a lack of care and attention experienced by highly spaced children. The short birth intervals portrayed by the data can also be a result of the low uptake of modern family planning methods.

Children who live in households with better toilet facilities have a decreased mortality rate. This finding is consistent with the results of the Centers for Disease Control and Prevention, which estimates that around 801,000 children under the age of five die each year from diarrhea as a result of poor sanitary conditions, predominantly in developing countries.

Children born in the Nyanza and Western regions were more likely than those born in the Central region to die before reaching the age of five. This result could potentially be explained by the uneven distribution of socioeconomic resources among regions. Furthermore, because of the Central region's proximity to Nairobi city, which has better health care systems, mothers in the Central region can easily access 
quality health care services, increasing survival chances of their children than those in the Western and Nyanza regions.

Unexpectedly, the data shows that sex of a child has no effect on under-five child mortality. This contradicts the findings of other similar researches [21]. The risk of under-five mortality is lowered if a mother is more educated (high school and above). This finding is consistent with other results of other studies [22], as a well-educated mother is more knowledgeable and capable of making well-informed decisions about healthcare services [23], such as vaccinations, which may improve a child's survival chances. A well-educated mother is also more likely to live in an economically developed location with improved hygiene conditions and better healthcare system, which will boost the survival chances of her children [24].

\section{Conclusion and Recommendation}

According to the study, under-five child mortality is still a problem in Kenya, and the findings should inform decision-makers prioritize reproductive, maternal, newborn, and child health care in national and sub-national budgets.

The region, place of residence, family size, total number of children in a household, mother's level of education, mother's occupation, birth order, and type of toilet facility were all found to be strongly associated with the U5CM rate in Kenya

To attain the Sustainable Development Goals by 2030, more intensive and effective interventions should be implemented to address these factors.

Targeted interventions aimed at empowering women and boosting health knowledge among mothers, especially in rural and lesser inaccessible areas, should be scaled up to improve health awareness and health service utilization and ultimately lower under-five child mortality rate in Kenya. The central government should implement socio-economic development interventions aimed at reducing regional disparities. National and subnational governments should commit resources to guarantee that modern contraceptives are available and utilized to increase child spacing, which will ultimately help reduce the $\mathrm{U} 5 \mathrm{CM}$ in the country.

In addition, more measures to enhance sanitation and hygiene, particularly in rural and less accessible areas, are required. Child survival may be impacted further by strategies targeted at ensuring equal regional socioeconomic development. More research into health-related factors that influence child mortality is required to inform relevant approaches and interventions for the survival of children in Kenya.

\section{Acknowledgements}

The completion of this research project was made possible through the help of many individuals who gave their support. I thank the Almighty God for granting me the knowledge to carry out this study. I can't overlook the love and support from my family and friends who encouraged me throughout the project. Dr. Anthony Wanjoya, my supervisor, deserves special thanks for his scholarly and professional help, as well as his presence to correct my work. He provided me with invaluable moral and academic support, which I greatly appreciate.

\section{References}

[1] Bay G, Miller T, Faijer DJ. WHO: Levels and trends in child mortality. Report 2013. 2013; 30.

[2] Hill K, Bicego G. Childhood Mortality in Kenya: An Examination of Trends and Determinants in the Late 1980s to Mid 1990s.

[3] Hamel MJ, Adazu K, Obor D, et al. A reversal in reductions of child mortality in Western Kenya, 2003-2009. Am J Trop Med Hyg [Internet]. The American Society of Tropical Medicine and Hygiene; 2011 [cited 2021 Jun 10]; 85 (4): 597-605. Available from: /pmc/articles/PMC3183762/.

[4] Schultz TP. Studying the impact of household economic and community variables on child mortality. Child Surviv Strateg Res. Cambridge University Press; Population \& Development Review, supplement to v. 10; 1984: 215-235.

[5] Mosley WH, Chen LC. An analytical framework for the study of child survival in developing countries. Child Surviv Strateg Res. Cambridge University Press; Population \& Development Review, supplement to vol. 10; 1984: 25-45.

[6] Rutstein SO. Factors associated with trends in infant and child mortality in developing countries during the 1990s [Internet]. Bull. World Health Organ. 2000. Available from: http://www.who.int/bulletin/tableofcontents/.

[7] Davanzo J, Razzaque A, Rahman M, et al. The Effects of Birth Spacing on Infant and Child Mortality, Pregnancy Outcomes, and Maternal Morbidity and Mortality in Matlab, Bangladesh THE EFFECTS OF BIRTH SPACING ON INFANT AND CHILD MORTALITY, PREGNANCY OUTCOMES, AND MATERNAL MORBIDITY AND MORTALITY IN MATLAB, BANGLADESH*. 2004.

[8] Kibet MK. Comparative study of infant and child mortality: The case of Kenya and South Africa. African Popul Stud [Internet]. Stellenbosch University; 2013 [cited 2021 Jun 10]; 24 (1-2): 1-2. Available from: http://aps.journals.ac.za.

[9] Rutstein SO. Effects of preceding birth intervals on neonatal, infant and under-five years mortality and nutritional status in developing countries: Evidence from the demographic and health surveys. Int J Gynecol Obstet [Internet]. John Wiley and Sons Ltd; 2005 [cited 2021 Jun 10]; 89 (SUPPL. 1): S7-S24. Available from: https://obgyn.onlinelibrary.wiley.com/doi/full/10.1016/j.ijgo.2 004.11.012.

[10] Kumar P, File G. Infant and child mortality in Ethiopia: A statistical analysis approach. Ethiop J Educ Sci [Internet]. African Journals Online (AJOL); 2011 [cited 2021 Jun 10]; 5 (2). Available from: https://www.ajol.info/index.php/ejesc/article/view/65373.

[11] Mutunga CJ. Environmental Determinants of Child Mortality in Kenya. Heal Inequal Dev [Internet]. Palgrave Macmillan UK; 2011 [cited 2021 Jun 10]. p. 89-110. Available from: https://link.springer.com/chapter/10.1057/9780230304673_5. 
[12] Wardlaw T, Jensen L, Moreira Daniels webSite L, Cassidy S, Yuen D, Rao Gupta G. Committing to Child Survival: A Promise Renewed UNICEF Country Offices contributed to the review of country example text. Policy and communications advice and support were provided by [Internet]. 2012 [cited 2021 Jun 10]. Available from: www.apromiserenewed.org.

[13] Hobcraft JN, Mcdonald JW, Rutstein SO. Socio-economic factors in Infant and child mortality: A cross-national comparison. Popul Stud (NY). 1984; 38 (2): 193-223.

[14] Stallings RY. Child morbidity and treatment patterns [Internet]. 2004 Dec. Available from: http://www.measuredhs.com.

[15] Ngome E. Demographic, Socio-Economic and Psychosocial Determinants of Current and Consistent Condom Use among Adolescents in Botswana. World J AIDS. Scientific Research Publishing, Inc,; 2016; 06 (04): 137-156.

[16] Mutunga CJ. A Service of zbw Environmental determinants of child mortality in Kenya Standard-Nutzungsbedingungen [Internet]. [cited 2021 Jun 10]. Available from: www.econstor.eu.

[17] Uaps 2007 [Internet]. [cited 2021 Jun 10]. Available from: https://uaps2007.princeton.edu/abstracts/70670.

[18] Fayehun OA. Household Environmental Health Hazards and Child Survival in Sub-Saharan Africa [WP74] [Internet]. 2010. Available from: www.measuredhs.com.

[19] Buttenheim AM. The sanitation environment in urban slums: Implications for child health. Popul Environ. 2008; 30 (1-2): 26-47.

[20] Ilinca S, Giorgio L Di, Salari P, Chuma J. Socio-economic inequality and inequity in use of health care services in Kenya: Evidence from the fourth Kenya household health expenditure and utilization survey [Internet]. Int. J. Equity Health. BioMed Central Ltd.; 2019 [cited 2021 Jun 10]. p. 1-13. Available from: https://doi.org/10.1186/s12939-019-1106-z.

[21] Malderen C Van, Oyen H Van, Speybroeck N. Contributing determinants of overall and wealth-related inequality in under-5 mortality in 13 African countries. J Epidemiol Community Health. 2013; 67 (8): 667-676.
[22] Nasejje JB, Mwambi HG, Achia TNO. Understanding the determinants of under-five child mortality in Uganda including the estimation of unobserved household and community effects using both frequentist and Bayesian survival analysis approaches Biostatistics and methods. BMC Public Health [Internet]. BioMed Central Ltd.; 2015 [cited 2021 Jun 10]; 15 (1): 1-12. Available from: https://bmcpublichealth.biomedcentral.com/articles/10.1186/s12889 $-015-2332-\mathrm{y}$.

[23] Basu AM, Stephenson R. Low levels of maternal education and the proximate determinants of childhood mortality: A little learning is not a dangerous thing. Soc Sci Med [Internet]. Elsevier Ltd; 2005 [cited 2021 Jun 10]; 60 (9): 2011-2023. Available from: https://pubmed.ncbi.nlm.nih.gov/15743650/.

[24] Ezeh OK, Agho KE, Dibley MJ, Hall J, Page AN. Determinants of neonatal mortality in Nigeria: Evidence from the 2008 demographic and health survey. BMC Public Health [Internet]. BioMed Central Ltd.; 2014 [cited 2021 Jun 10]; 14 (1): 1-10. Available http://www.biomedcentral.com/1471-2458/14/521.

\section{Biography}

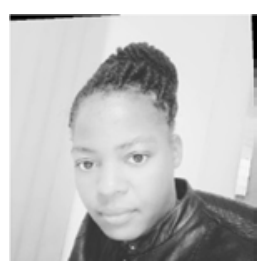

Elda Naliaka Watulo is a statistician with expertise in statistical data analysis, database development and management, and report writing. She has worked with AMREF Health Africa, Kenya and Deutsche Stiftung Weltbevölkerung (DSW) as a data, monitoring and evaluation specialist. She has practical experience in designing and conducting impact evaluations, conducting both qualitative and quantitative data analysis using SPSS, STATA, R, Advanced MS Excel, and Nvivo. She has published several papers in the Journal of Water, Sanitation \& Hygiene for Development, and others. 